\title{
Clustering and conductance in breakage of sodium nanowires
}

\author{
A. Zugarramurdi, ${ }^{1,2}$ A. G. Borisov, ${ }^{3}$ N. Zabala,,${ }^{1,2,4}$ E. V. Chulkov, ${ }^{2,4,5}$ and M. J. Puska ${ }^{6}$ \\ ${ }^{1}$ Elektrizitatea eta Elektronika Saila, Zientzia eta Teknologia Fakultatea, UPV/EHU, 644 P.K., 48080 Bilbao, Spain \\ ${ }^{2}$ Centro de Física de Materiales (CFM)-Materials Physics Center (MPC), Centro Mixto CSIC-UPV/EHU, \\ 20018 San Sebastian/Donostia, Spain \\ ${ }^{3}$ CNRS, Institut des Sciences Moléculaires d'Orsay, ISMO, UMR 8214 CNRS-Université Paris-Sud, \\ Bâtiment 351, Université Paris-Sud, 91405 Orsay CEDEX, France \\ ${ }^{4}$ Donostia International Physics Center (DIPC), P. de Manuel Lardizabal 4, 20018 San Sebastián/Donostia, Spain \\ ${ }^{5}$ Departamento de Física de Materiales, Facultad de Ciencias Químicas, Universidad del País Vasco UPV/EHU, Apartado 1072, \\ 20080 San Sebastián/Donostia, Spain \\ ${ }^{6}$ Department of Applied Physics, Aalto University, P. O. Box 11100, FIN-00076 AALTO, Finland \\ (Received 5 May 2010; revised manuscript received 28 June 2010; published 10 January 2011)
}

\begin{abstract}
We study the conductance during the elongation and breakage of Na nanowires described with the ultimate jellium model. A combined approach is used where the nanowire breakage is simulated self-consistently within the density-functional theory, and the wave packet propagation technique is applied for ballistic electron transport. For certain conditions the breakage of the nanowire is preceded by formation of clusters of magic size in the break junction. This affects the conductance $G$, in particular the shape of the $G=3 G_{0}$ to $G=G_{0}\left(=2 e^{2} / h\right)$ step upon elongation. The observed trends can be explained as due to the transient trapping of ballistic electrons inside the cluster, leading to a resonant character of the electron transport through the break junction. The cluster-derived resonances appear as peak structures in the differential conductance which may serve as an experimental signature of clustering.
\end{abstract}

DOI: 10.1103/PhysRevB.83.035402

PACS number(s): 73.40.Jn, 73.23.Ad, 73.63.Nm, 73.63.Rt

\section{INTRODUCTION}

Owing to fundamental and practical interest, metallic nanowires are a subject of considerable experimental and theoretical effort. ${ }^{1}$ Not only is the nanowire a natural component of molecular electronics devices, but it can also serve as an efficient chemical and biological sensor. ${ }^{2}$ From the fundamental point of view, when drawn to the atomic dimension, nanowires exhibit quantum conductance behavior, ${ }^{3-5}$ thus offering an excellent playground for theoretical developments. Nowadays, systematic studies of electronic and mechanical properties of nanocontacts are possible thanks to the mechanically controllable break junction (MCBJ) and scanning tunneling microscopy experiments. ${ }^{1,6}$ In particular, MCBJ experiments are very well suited for conductance measurements, providing histograms for large sets of individual contact-breaking events as the basic input for further analysis. For simple metals, the conductance curves upon stretching show clear plateaus at values $n G_{0}=n 2 e^{2} / h$ ( $n$ is an integer) followed by more or less abrupt jumps to lower conductance plateaus. ${ }^{7,8}$ The appearance of plateaus is considered as a fingerprint of a stable nanowire structure in the contact neck, whereas abrupt conductance jumps are associated with instabilities and consequent sudden rearrangements of the neck. Thus, the electronic (atomic) shell structures of nanowires could be demonstrated with this technique ${ }^{3,4,9}$ where the stable nanowire configurations are characterized by "magic radii" in analogy to the well-known "magic numbers" of abundance in metal clusters of different sizes. ${ }^{10}$

Although great progress has been made in observing new structures in high-resolution transmission electron microscopy, ${ }^{5,11}$ the structural details of nanowires and their dynamical properties are far from being well understood. From the theoretical side, different approaches have been used to elucidate the atomistic rearrangements taking place during the breakage of a nanowire. Classical and semiclassical methodologies have been used along with $a b$ initio calculations based on density-functional theory (DFT). ${ }^{12-28}$ The latter, in particular when combined with electronic transport calculations, ${ }^{20-23}$ provides the most advanced description of the system, allowing direct connection with experimental data. However, DFT-based calculations are computationally extremely demanding, which severely limits the number of atoms (the size of the break junction) that can be addressed. Molecular dynamics (MD) remains then as a useful alternative if one wants to explore large sets of possible configurations. $^{24}$

Another way of tackling the complex problem of the evolution of the geometry and conductance of stretched nanowires is to reduce the level of complexity in description of the atomic structure of the system and to use DFT methodology, but based on jellium models for the nanowire material. Despite its apparent simplicity, such an approach can often capture the main physics of the process at hand, as has been proven with many studies of metal nanostructures and metal surfaces ${ }^{29}$ including the representation of electrodes in conductance studies. ${ }^{30}$ Among metallic nanowires sodium is the prototype system for the jellium description, allowing one to efficiently explore various structures. ${ }^{1,31,32}$ The nearly free character of sodium valence electrons fully validates the applicability of the jellium approach as shown in a number of works ${ }^{31-35}$ based on comparisons with ab initio studies and experimental data (see also Fig. 2 of this paper). Thus, in full agreement with first-principles molecular dynamics simulations, ${ }^{24-26}$ Ogando et al. reported the formation of clusterlike arrangements of atoms, preceding the breakage of a stretched nanowire. ${ }^{35}$ In that work the ultimate jellium (UJ) 


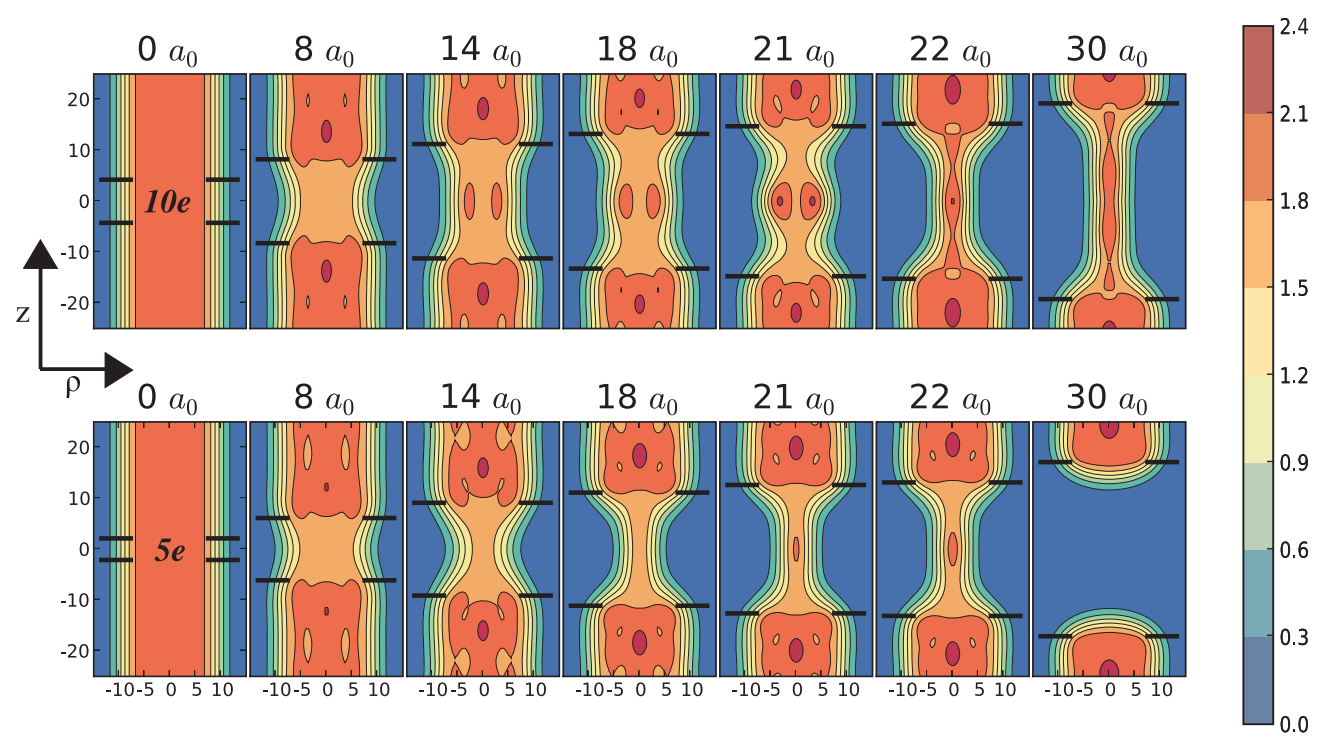

FIG. 1. (Color online) Snapshots of the charge density for elongations $\Delta L=0,8 a_{0}, 14 a_{0}, 18 a_{0}, 21 a_{0}, 22 a_{0}$, and $30 a_{0}$ during the stretching of a nanowire of magic radius $10.7 a_{0}$. The charge density values in the color bar are multiplied by a factor of 1000 . The figures at the top (bottom) correspond to the simulations with ten (five) electrons initially in the deformable constriction. The deformable constriction is delimited by the horizontal marks. The $10 e$ system is broken at $\Delta L=31 a_{0}$.

model, first used by Manninen for clusters, ${ }^{36}$ was applied. It is worth mentioning that as far as closed-shell sodium clusters are concerned, the jellium model gives adequate description of many physical properties. ${ }^{37}$ As for the nanowire breakage, the UJ approach can be interpreted as giving directly some kind of average configuration, so that large MD statistics is not needed.

Here we report on how the conductance of the elongated nanowire is affected by the formation of stable clusterlike arrangements of atoms in the break junction. We find that clustering leads to delayed and rounded conduction jumps upon the stretching of the nanowire. The results are analyzed in terms of the evolution of the transmission resonances originating from the cluster-localized electronic states. The situation at hand then closely corresponds to the electron transport through molecular junctions studied in great detail in the context of molecular electronics. ${ }^{1,38-43}$ The cluster is playing the role of the molecular object. According to our results the resonant character of the transmission reveals itself particularly clearly in the bias voltage dependence of the differential conductance, suggesting experimental ways to evidence the presence of clusters in the break junction. It should be pointed out that the main conclusions and results reported in this work are not determined by the specific choice of the nanowire material $(\mathrm{Na})$ but rather by the very fact of the clustering at the break junction. The latter phenomenon is of quite general nature, as it not only happens for the stretched Na nanowires ${ }^{24-26,35}$ but has also been reported for gold nanowires. ${ }^{23,27,28}$

The present paper is organized as follows. Section II introduces the system under study and presents a brief outline of the theoretical methods. Section III is devoted to the results and their discussion. Section IV presents the summary and conclusions. Atomic units are used throughout the paper unless otherwise stated.

\section{METHODS}

We use a combined approach where the nanowire breakage is simulated with self-consistent electronic structure calculations within the density-functional theory. ${ }^{44}$ The selfconsistent calculations provide an input for the follow-up wave packet propagation (WPP) study of the ballistic electron transport through the break junction.

The DFT calculations are based on the UJ description of nanowires. ${ }^{36}$ Within the UJ model the background positive charge is fully relaxed in shape and density, so that it equals at every point the electron density, and the shape of the system is obtained self-consistently. In this way, it is possible to mimic the rearrangements of atoms during the elongation and breakage of a nanowire with MCBJ techniques, for example. The UJ model is limited to a unique equilibrium charge density with the density parameter $r_{s} \approx 4.2 a_{0}$ [the density $\left.n=3 /\left(4 \pi r_{s}^{3}\right)\right]$. This value corresponds approximately to sodium metal. A nice property of this particular model for nanowires is that UJ always creates a force which opposes the elongation due to an external force. ${ }^{35}$ This is in accordance with experiments and with atomistic simulations but it is not found in other jellium models.

The implementation of the present approach is explained in a previous paper, ${ }^{35}$ so we just comment on its most important features. The system considered in the simulations is sketched in Fig. 1. It consists of two cylindrical UJ leads (we restrict the shapes of the nanowires to those having axial symmetry), whose potential is frozen after self-consistent calculations for an infinite wire of stable magic radius $R$. Rearrangements are only allowed in the central part (break junction) between the leads, which is deformable upon stretching of the nanowire. The shape in this region is updated in a self-consistent process. Setting the size $L$ of this part allows one to define the number of electrons $N_{\bar{e}}$ in the deformable constriction. This is a key quantity as it determines whether clusterlike structures 
are or are not formed in break junctions. ${ }^{35}$ We note that the procedure of constraining the number of electrons in the deformable part is equivalent to the usual methodology followed in $a b$ initio approaches, where only some atoms are allowed to relax between the frozen leads. The results reported below are obtained for the breakage of a nanowire of radius $R=10.7 a_{0}$. This is a typical example of a stable $\mathrm{Na}$ nanowire structure allowing one to study the change of the conductance both in the presence and in the absence of cluster-derived structures in the deformable constriction during the stretching process. Concerning the computational methods, DFT Kohn-Sham equations are solved numerically and self-consistently in the cylindrical real space $(\rho, z)$ using a multigrid method. ${ }^{45}$ For each elongation $\Delta L$ a self-consistent effective potential $V(\rho, z)$ is calculated.

The effective one-electron potential obtained in the DFT study is then used in the WPP calculation of the evolution of the conductance curves upon elongation. The details of the WPP technique can be found elsewhere. ${ }^{46,47}$ Here we only address the aspects specific for the present study. In brief, the one-electron wave packet describing the ballistic electron is propagated through the deformable constriction by solving the time-dependent Schrödinger equation (TDSE). Because of the symmetry of the problem the electronic wave function is represented on a grid in cylindrical coordinates:

$$
\Psi(\rho, \varphi, z, t)=\sum_{m} \psi_{m}(\rho, z, t) e^{i m \varphi}
$$

The $m$ projection of the angular momentum on the quantization $z$ axis is a good quantum number, so that different $m$ subspaces are treated independently. The time evolution of $\psi_{m}(\rho, z, t)$ is governed by the TDSE

$$
i \partial_{t} \psi_{m}(\rho, z, t)=H_{m} \psi_{m}(\rho, z, t)
$$

with the Hamiltonian

$$
H_{m}=-\frac{1}{2} \frac{\partial^{2}}{\partial z^{2}}-\frac{1}{2 \rho} \frac{\partial}{\partial \rho} \rho \frac{\partial}{\partial \rho}+\frac{m^{2}}{2 \rho^{2}}+V(\rho, z)+V_{a b s}(z) \text {. }
$$

In Eq. (3) $V_{a b s}$ is an absorbing potential ${ }^{48,49}$ introduced at the $z$ extremities of the mesh to avoid the artificial reflections of the wave packet. Provided initial conditions $\psi_{m}(\rho, z, t=0)$, Eq. (2) is solved via short-time propagation with the splitoperator technique ${ }^{50,51}$ as detailed in Refs. 46,47.

In the asymptotic regions far from the deformable constriction the nanowire potential is a function of only the $\rho$ coordinate: $V=V(\rho)$. The electron confinement in the $\rho$ direction perpendicular to the nanowire axis leads then to a series of quantized states $\phi_{n}^{m}(\rho)$ with energies $E_{n}^{m}$ :

$$
\left[-\frac{1}{2 \rho} \frac{\partial}{\partial \rho} \rho \frac{\partial}{\partial \rho}+\frac{m^{2}}{2 \rho^{2}}+V(\rho)\right] \phi_{n}^{m}(\rho)=E_{n}^{m} \phi_{n}^{m}(\rho) .
$$

In Tables I and II we report on the energies $E_{n}^{m}$ of the transverse states obtained in the present calculations for the two nanowire radii of $10.7 a_{0}$ and $7.7 a_{0}$. The second case appears useful for the discussion on the evolution of the conductance under the stretching process (see the next section). The energy is given with respect to the Fermi level of the undistorted Na nanowire. The $\pm m$ states are degenerate.
TABLE I. Transverse eigenenergies $E_{n}^{m}$ for the $R=10.7 a_{0} \mathrm{Na}$ nanowire (up to $1.5 \mathrm{eV}$ ). The results are given in $\mathrm{eV}$ with respect to the Fermi level.

\begin{tabular}{lrrr}
\hline \hline & $n=1$ & $n=2$ & $n=3$ \\
\hline$m=0$ & -2.58 & -0.62 & 1.28 \\
$|m|=1$ & -1.75 & 0.42 & \\
$|m|=2$ & -0.79 & 1.30 & \\
$|m|=3$ & 0.25 & & \\
$|m|=4$ & 1.30 & & \\
\hline \hline
\end{tabular}

In order to obtain the conductance of the system, one is interested in the energy-dependent transmission $T_{n n^{\prime}}^{m}(E)$ and reflection $R_{n n^{\prime}}^{m}(E)$ coefficients describing the ballistic electron transport through the deformable constriction. The labels $n$ and $n^{\prime}$ refer here to the asymptotic initial (incident) and final (reflected or transmitted) channels, respectively. In the discussion below, for the simplicity of the presentation we will also use the $(m, n)$ labeling of the channels. An asymptotic channel is described by the wave function

$$
\Phi_{n}^{m}(\rho, z)=\frac{1}{\sqrt{2 \pi}} e^{i k z} \phi_{n}^{m}(\rho) .
$$

It corresponds to an electron of a given energy $E=E_{n}^{m}+k^{2} / 2$ propagating along the nanowire in the $z$ direction and confined in the $\rho$ direction. The channel is open when $E>E_{n}^{m}$.

The $T_{n n^{\prime}}^{m}(E)$ and $R_{n n^{\prime}}^{m}(E)$ coefficients are calculated within each $m$ subspace from the time-dependent solution $\psi_{m}(\rho, z, t)$ using the virtual detector method. ${ }^{52}$ To this end, the initial state $\psi_{m}(\rho, z, t=0)$ is defined as follows:

$$
\psi_{m}(\rho, z, t=0)=\phi_{n}^{m}(\rho) e^{i k_{0} z-\left(z-z_{0}\right)^{2} / \sigma^{2}},
$$

where the parameters $k_{0}$ and $\sigma$ are chosen in such a way that (i) the initial wave packet has no overlap with the deformable constriction, and it is composed of only incident waves, and (ii) the energy spectrum of the initial wave packet covers the energy range of interest. We have carefully checked that flux is conserved, i.e., $\sum_{n^{\prime}}\left[T_{n n^{\prime}}^{m}(E)+R_{n n^{\prime}}^{m}(E)\right]=1$ is fulfilled in the energy region of interest. The summation here runs over the open channels.

Finally, the zero-bias conductance is obtained with the Landauer-Büttiker formula ${ }^{53}$

$$
G=G_{0} \sum_{m, n, n^{\prime}} T_{n n^{\prime}}^{m}\left(E=E_{F}\right),
$$

where $E_{F}$ stands for the Fermi level energy, and the summation runs over the open channels. From Table I it follows that for the nonstretched perfect $10 e$ nanowire $G=6 G_{0}$ and it is determined by the $(m=0, n=1),(m=0, n=2)$, and doubly

TABLE II. Transverse eigenenergies $E_{n}^{m}$ for the $R=7.7 a_{0} \mathrm{Na}$ nanowire (up to $1.5 \mathrm{eV}$ ). The results are given in $\mathrm{eV}$ with respect to the Fermi level.

\begin{tabular}{lrc}
\hline \hline & $n=1$ & $n=2$ \\
\hline$m=0$ & -2.30 & 0.39 \\
$|m|=1$ & -1.01 & 1.36 \\
$|m|=2$ & 0.34 & \\
\hline \hline
\end{tabular}


degenerate $(m= \pm 1, n=1)$, and $(m= \pm 2, n=1)$ channels. Indeed, for the perfect wire $T_{n n^{\prime}}^{m}=\delta_{n n^{\prime}}$ with $\delta_{n n^{\prime}}$ being the Kronecker delta symbol.

\section{RESULTS AND DISCUSSION}

\section{A. Conductance during nanowire breaking}

We found that for the nanowire of radius $R=10.7 a_{0}$, at a minimum, seven initial electrons in the deformable constriction are needed to enable the appearance of the eight-electron cluster structures in the break junction. The "extra" electron density is supplied in this case by the leads. In Fig. 1, we represent the two different evolutions of the charge density of the systems with $N_{\bar{e}}=5$ and $N_{\bar{e}}=10$ electrons in the corresponding initial deformable constrictions, which will be used as representative examples throughout this work (we will refer to these systems as $5 e$ and $10 e$, respectively). Two fundamentally different breakage patterns are observed with the appearance of cluster-derived structures in the $10 e$ system at elongations from $14 a_{0}$ to $21 a_{0}$, just before the "monatomic chain" configuration stage. In what follows we shall focus on the consequences of these two breakage patterns on the conductance curves.

The calculated change in conductance during the nanowire stretching is shown in Fig. 2 for $5 e$ and $10 e$ prototype breakages (see Fig. 1). It follows from the present results that, at the initial stages of the elongation, the conductance change from $6 G_{0}$ to $3 G_{0}$ happens in a similar way in both systems. As well, inspection of Figs. 2 and 1 shows that the final $G=G_{0}$ plateau is related in both systems to the existence of the monatomic chain supported between metal leads. However, two different behaviors are distinguished in the change from the $G=3 G_{0}$ to the $G=G_{0}$ plateau. Specifically, in the breakage of the $10 e$ system the conductance $G \simeq 3 G_{0}$ is maintained over a much broader range of nanowire elongations as compared to the $5 e$ case. As follows from Fig. 1, this is exactly the region of the elongations where clustering happens for the $10 e$ system. We thus tentatively attribute the difference in the conductance change from $3 G_{0}$ to $G_{0}$ for the $5 e$ and $10 e$ systems to the

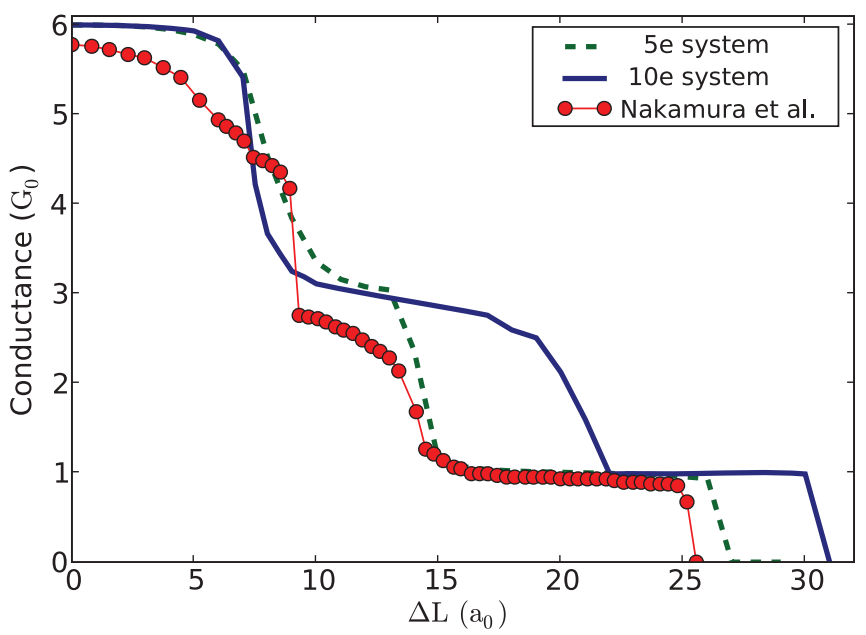

FIG. 2. (Color online) Calculated conductances as a function of the nanowire elongation $\Delta L$. The results for $10 e$ and $5 e$ systems are shown together with ab initio data by Nakamura et al. (Ref. 20). formation of a cluster structure in the break junction in the $10 e$ case. The (red) dots in Fig. 2 show the results of $a b$ initio calculations performed by Nakamura et $a l .{ }^{20}$ within the supercell geometry. Because of the limited size of the supercell no clustering was reported in Ref. 20. The $a b$ initio and present $5 e$ results are in agreement with respect to the nanowire elongations corresponding to the conductance steps and the conductance values at the plateaus which reflect the quasidegeneracy of the transverse eigenstates. This shows that the jellium model allows adequate description of the system. We will return to the comparison between the present and $a b$ initio calculations at the end of this section.

\section{B. Conductance channels}

To get further insight into the calculated trends we present in Fig. 3 the decomposition of the conductance curve into different $(m, n)$ incident channels defined as

$$
G_{n}^{|m|}=\alpha_{|m|} G_{0} \sum_{n^{\prime}} T_{n n^{\prime}}^{|m|}\left(E=E_{F}\right) .
$$

The transmission probabilities are summed over all possible final states so that $G_{n}^{|m|}$ represents the contribution of the $n$th incident channel within $\pm m$ symmetry to the total conductance. The $\alpha_{|m|}$ coefficient ( 1 for $m=0$, and 2 otherwise) accounts for the degeneracy of the $\pm m$ states.

As follows from Fig. 3, the initial decrease of the conductance from $6 G_{0}$ to $3 G_{0}$ for the nanowire elongation $\Delta L=8 a_{0}$ is linked with the drop of the transmission for the ( $m=0, n=$ 2 ) and doubly degenerate $(m= \pm 2, n=1)$ incident channels in both $10 e$ and $5 e$ cases. For this elongation range the break junction forms a neck which can be approximately described as a portion of the nanowire of magic radius $R=7.7 a_{0}$ smoothly attached to the $R=10.7 a_{0}$ leads. Assuming the adiabatic approximation $^{1}$ to be valid in the present case (i.e., that the transverse quantum number $n$ is preserved upon propagation through the junction), from Tables I and II one concludes that transmission at the Fermi energy is only possible for

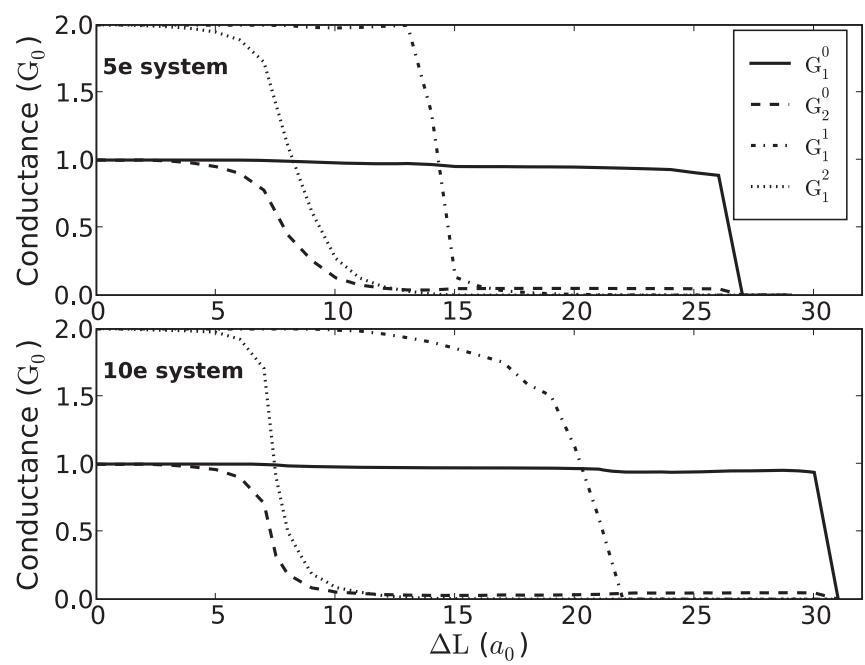

FIG. 3. Contributions of different incident channels to the total conductance $G_{n}^{|m|}$ [see Eq. (8)] as a function of the nanowire elongation $\Delta L$. The upper (lower) panel corresponds to the simulations with five (ten) electrons initially in the deformable constriction. 
the $(m=0, n=1)$ and $(m= \pm 1, n=1)$ incident channels. The $E_{2}^{0}$ and $E_{1}^{ \pm 2}$ energies rise well above the Fermi level for the $R=7.7 a_{0}$ nanowire, forming an efficient tunneling barrier for electrons arriving from the $R=10.7 a_{0}$ leads. The corresponding channels are then closed.

The validity of the adiabatic approximation in the present case stems from the nearly diagonal structure of the calculated transmission matrix $T_{n n^{\prime}}^{|m|}(E)$. In this respect the $(m, n)$ channels closely correspond to the conductance eigenchannels. ${ }^{1,54,55}$ However, the adiabatic approximation breaks down for values of the electron energy or the nanowire elongation for which the overall transmission for the given incident channel becomes small. Thus, e.g., the ( $m=0, n=2)$ channel is never extinguished completely. $G_{2}^{0}$ reaches the $\sim 0.03 G_{0}$ minimum at $\Delta L \approx 15 a_{0}$ and during the following stretching it slightly increases to $\sim 0.05 G_{0}$ until complete breakage occurs. It follows from our result that for $\Delta L>14 a_{0} G_{2}^{0}$ is entirely given by the nondiagonal $T_{21}^{0}\left(E=E_{F}\right)$ matrix element. Thus, the transmission is due to the interchannel coupling between the $(m=0, n=2)$ and $(m=0, n=1)$ channels. In accordance with the principle of detailed balance and because of the mirror symmetry of the system, we also find that $T_{21}^{0}=T_{12}^{0}$, i.e., an electron incident at the break junction within the $(m=0, n=$ 1) channel has a nonzero probability to be transmitted into the ( $m=0, n=2$ ) subband.

The final $G=G_{0}$ conductance plateau is mainly due to the transmission within the incident $(m=0, n=1)$ channel [there is a small contribution of the $(m=0, n=2)$ channel as discussed above]. During this final stretching stage, a monatomic UJ wire of average radius $R=4.5 a_{0}$ is formed as shown in Fig. 1. This monatomic wire is broken at the elongation $\Delta L=27 a_{0}$ for the $5 e$ system and at $\Delta L=31 a_{0}$ for the $10 e$ system. During the monatomic configuration of the neck, the conductance is in the range of $0.98 G_{0}-1.0 G_{0}$, which is in good agreement with published $a b$ initio data for the smaller oscillation amplitudes with respect to the number of atoms in suspended monatomic chains. ${ }^{20,56,57}$

\section{The eight-electron cluster}

Let us now turn to the detailed discussion of the conductance curves in the $14 a_{0} \leqslant \Delta L \leqslant 21 a_{0}$ elongation range, where the clustering happens for the $10 e$ system (see Fig. 1). We argue that it is the formation of the cluster in the break junction for the $10 e$ system that is responsible for the shape of the conductance change from $G=3 G_{0}$ to $G=G_{0}$ as calculated in the present system. The cluster in the break junction appears particularly clear in the $\Delta L=21 a_{0}$ panel of Fig. 1. Analysis of the cluster charge from the calculated electronic density shows that it is close to 8 , i.e., the break junction represents the nearly spherical $8 e$ magic cluster smoothly attached to the $R=10.7 a_{0}$ leads.

The situation at hand then closely corresponds to the case of a molecule attached to two metal electrodes, the problem currently studied in the field of molecular electronics. ${ }^{1,39-43}$ Intuitively, the electron transmission through the junction should show resonant structures corresponding to the transient trapping of ballistic electrons inside the cluster. On the other hand, resonances can be seen as discrete cluster states which are broadened because of the coupling to the continua
TABLE III. Energies of the lowest-lying shells of the spherical free-standing $8 e \mathrm{UJ}$ cluster. The results are given in $\mathrm{eV}$ with respect to the Fermi level of the $R=10.7 a_{0}$ nanowire.

\begin{tabular}{lr}
\hline \hline Shell & Energy \\
\hline$s(m=0)$ & -1.93 \\
$p(m=0, \pm 1)$ & -0.57 \\
$d(m=0, \pm 1, \pm 2)$ & 0.81 \\
\hline \hline
\end{tabular}

of the propagating electronic states inside the leads. ${ }^{39,40}$ Table III lists the lowest energy levels of the free-standing spherical $8 e$ cluster as obtained with the present UJ jellium DFT calculations. The results are reported with respect to the Fermi level of the pristine $R=10.7 a_{0}$ nanowire. The occupied $s(\ell=0)$ and $p(\ell=1)$ shells and the nonoccupied $d(\ell=2)$ shell fall into the energy range relevant for electron transport through the break junction. Here, $\ell$ stands for the angular momentum.

In order to reveal the role of the resonances in the electron transport through the break junction we show in Fig. 4 the energy-resolved transmission probability defined for the given incident channel as follows:

$$
\mathbb{T}_{n}^{|m|}(E)=\sum_{n^{\prime}} T_{n n^{\prime}}^{|m|}(E),
$$

where the summation runs over all open final channels. Obviously $\alpha_{|m|} G_{0} \mathbb{T}_{n}^{|m|}\left(E_{F}\right)=G_{n}^{|m|}$, as given by Eq. (8).

For the $10 e$ constriction at the elongation $\Delta L=21 a_{0}$ one clearly observes a well-resolved low-energy resonant structure in the transmission within each $m$-symmetry subspace. The transmission reaches unity at the resonance as is well documented for molecular junctions, ${ }^{40,41,54}$ and can be demonstrated on the basis of the Lippmann-Schwinger equation in the case of the resonance-dominated transmission. ${ }^{58,59}$ Comparison with the data reported in Table III shows that the resonant energies correspond to the $s, p$, and $d$ shells of the free-standing $8 e$ cluster for the $m=0,1$, and 2 symmetries, respectively. For the $m=0$ symmetry data with the $(m=0, n=2)$ incident channel we also observe a $d$-shell resonance in $\mathbb{T}_{2}^{0}$. This is energetically degenerate with a resonant structure in $\mathbb{T}_{1}^{2}$ for the $m=2$ symmetry. Thus, the junction can indeed be seen as a spherical cluster attached to the leads. The resonant character of the electron transmission reflects, then, transient electron trapping inside the cluster.

Another valuable piece of information comes from the evolution of the transmission curve and, in particular, that of the resonances as a function of the elongation $\Delta L$. While the energy position is preserved through the sequence $14 a_{0}-18 a_{0}-21 a_{0}$, the width of the resonance decreases, i.e., the resonant structure sharpens [the minimum full width at half maximum (FWHM) is approximately $0.1 \mathrm{eV}$ for a Lorentzian fit]. This points at the change in the coupling between the cluster-localized states and leads. Comparison of the $\Delta L=14 a_{0}$ and $\Delta L=21 a_{0}$ panels of Fig. 1 shows that for $\Delta L=21 a_{0}$ the necks on each side of the cluster are much better defined. The cluster appears less strongly coupled to the leads, consistent with the smallest widths of the resonances in each $m$ subspace. Finally, the resonances vanish 

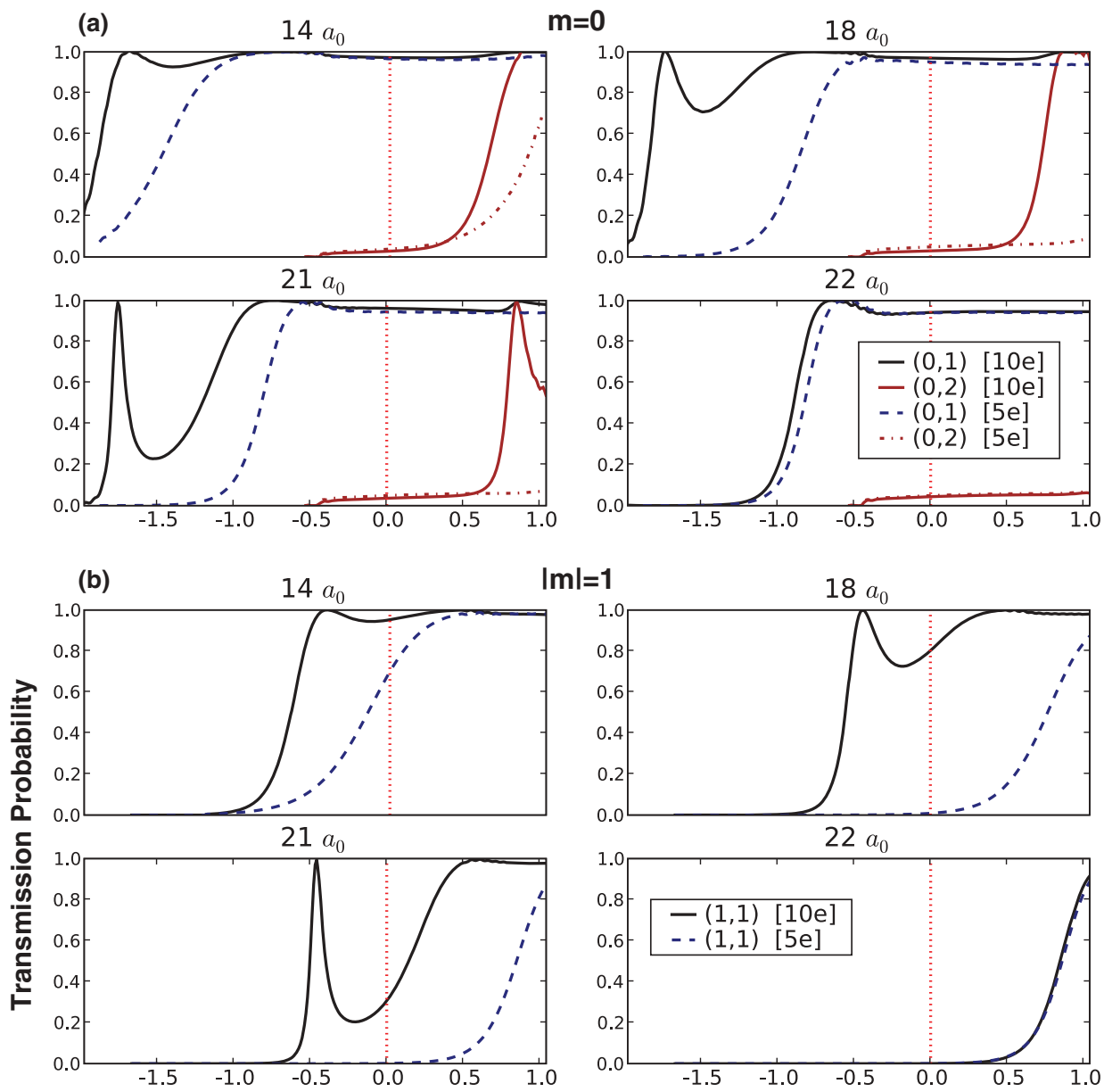

$\mid \mathrm{ml}=1$
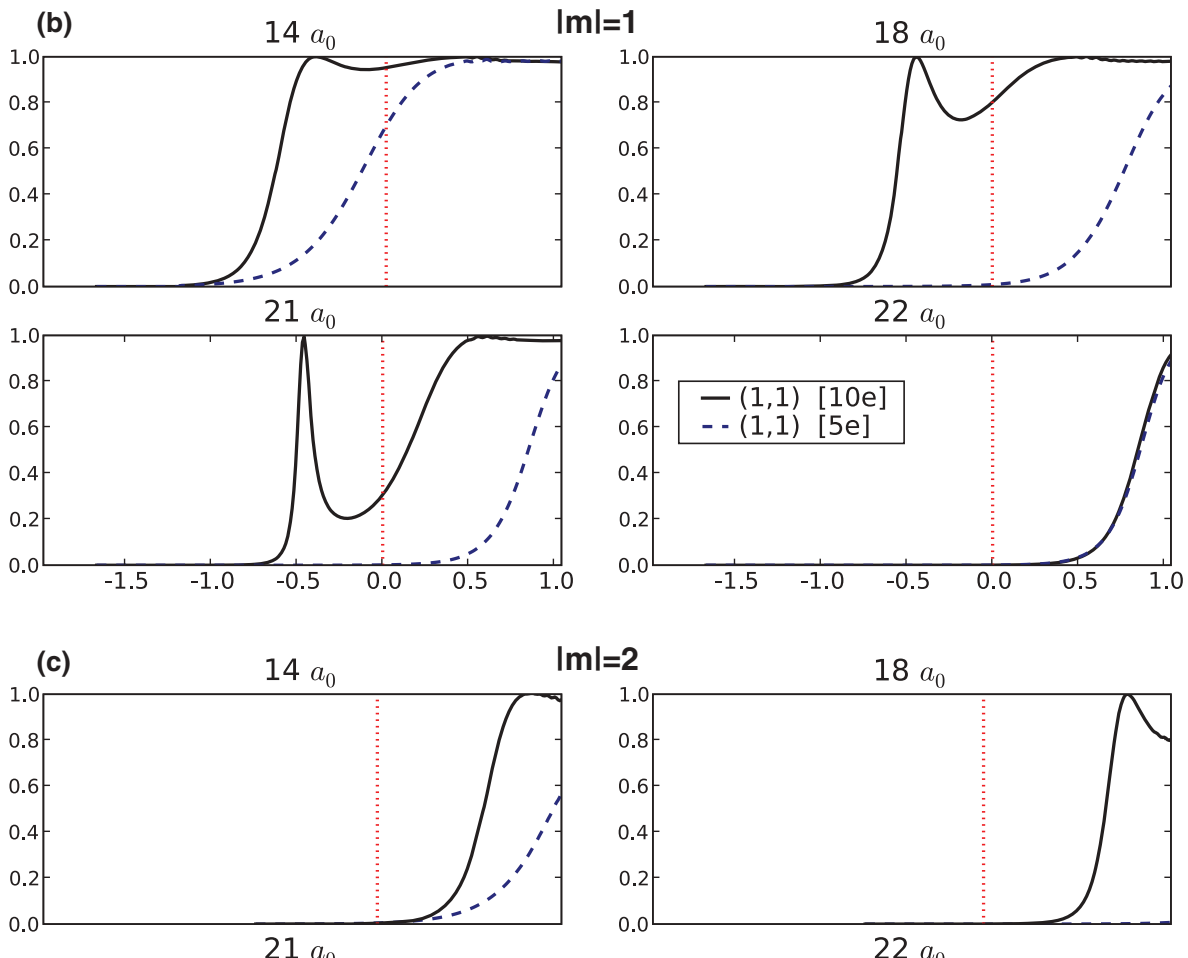

$\mathrm{ml}=\mathbf{2}$
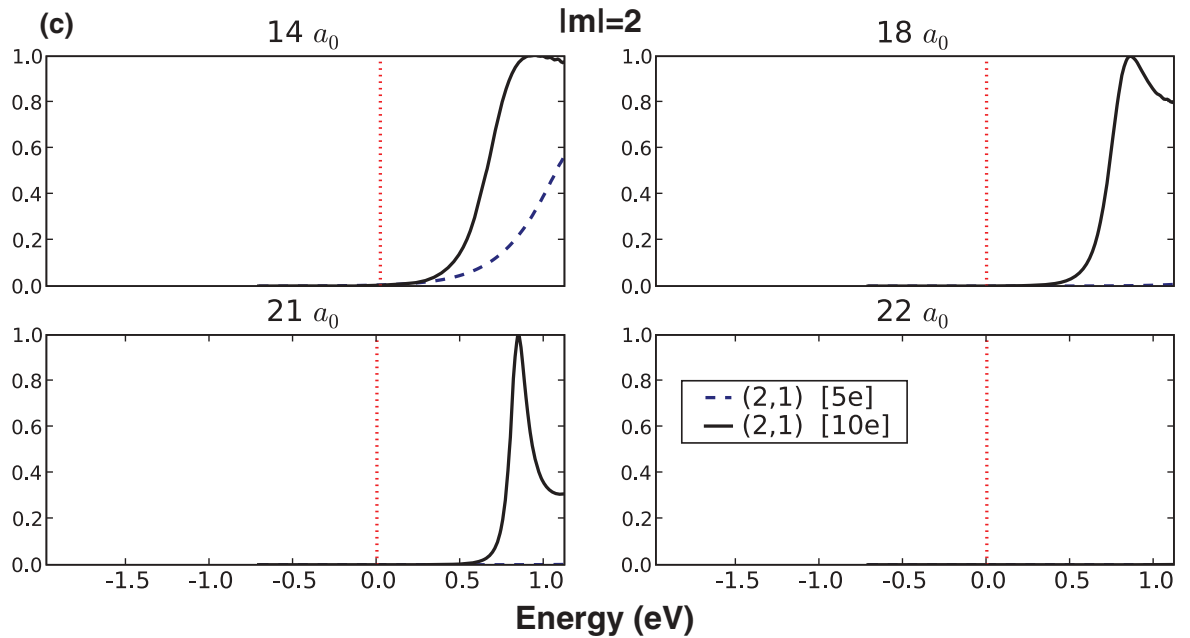

FIG. 4. (Color online) Transmission probability $\mathbb{T}_{n}^{|m|}(E)$ for different $(m, n)$ incident channels. Results are shown as a function of the energy of the incident electron for different elongations of the nanowire (defined above each panel). The energy is measured with respect to the Fermi level denoted by the vertical dotted line. The solid (dashed) lines correspond to the system with ten (five) electrons in the initial UJ deformable constriction. For further details, see the legends.

for $\Delta L=22 a_{0}$ when the $8 e$ cluster structure disappears from the break junction.

While the well-defined lowest-lying transmission resonance within each $m$-symmetry subspace can be unambiguously identified as the corresponding state of the free-standing cluster broadened by the electron coupling with the leads, the number of resonances observed in Fig. 4 is less than the number of states in the cluster. For example, the resonance at $-0.5 \mathrm{eV}$ observed within the $m=1$ symmetry emerges from the $p$ shell of the cluster and thus has to have its counterpart 

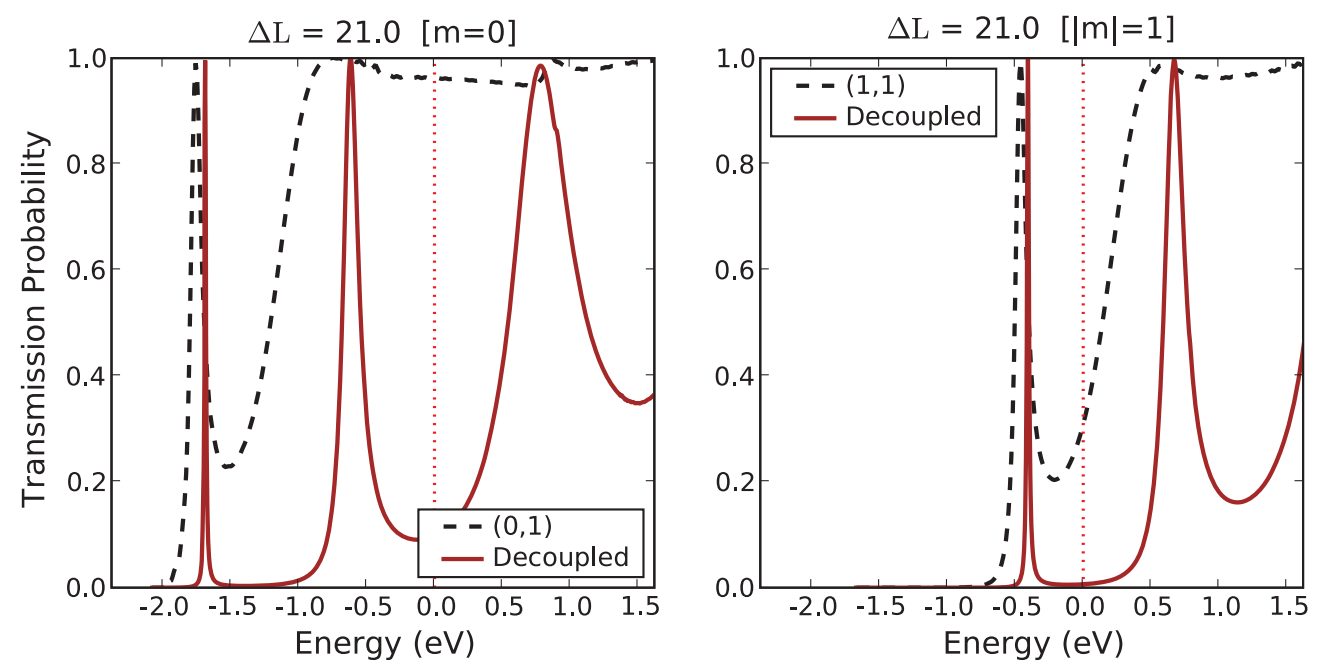

FIG. 5. (Color online) Transmission probability $\mathbb{T}_{n}^{|m|}(E)$ for the $(m=0, n=1)$ and $(m= \pm 1, n=1)$ incident channels for the $10 e$ case. The results are shown as a function of the energy of the incident electron measured with respect to the Fermi level, denoted by the vertical dotted lines. The calculations are performed for the nanowire elongation $\Delta L=21 a_{0}$. The dashed and solid lines correspond to the results obtained with the self-consistent potential and the potential with artificially increased reflectivity at the necks separating the cluster from the leads, respectively.

within the $m=0$ symmetry. However, it is not revealed by the calculation. Similarly, the resonance at $+0.8 \mathrm{eV}$ observed within the $m=2$ symmetry emerges from the $d$ shell of the spherical cluster. It thus has to appear within the $m=0$ and $m=1$ symmetry subspaces. This $d$-shell-derived resonance is indeed present for the $m=0$ symmetry, as follows from the $(m=0, n=2)$ transmission curve, $\mathbb{T}_{2}^{0}$. At the same time the $m=1$ symmetry results do not show a clear resonant structure within the corresponding energy range.

The "missing states" can be explained by a strong coupling between electronic states trapped in the cluster and those propagating in the leads. Potential barriers between the cluster and the leads are formed by the necks on each side of the cluster. Well-defined resonances in transmission appear when the energy of the underlying cluster state is below the potential barriers so that only resonant transmission is possible. For the energies above the potential barriers the resonant structures are broad. Moreover, the contrast for the resonance observation is much reduced since the nonresonant over-the-barrier transmission is high. ${ }^{39}$ To support this idea we have performed additional calculations where the reflectivity of the potential barrier between the cluster structure and the leads has been artificially increased. The self-consistent attractive potential in both necks between the cluster structure and the leads has been set to zero (i.e., to the vacuum level) in the regions with a width of $2 a_{0}$ in the $z$ coordinate around each neck.

Results of this calculation are reported in Fig. 5. All resonances expected from the shell structure of the freestanding cluster emerge. An important result follows from this calculation: both for the over-the-barrier and under-the-barrier character of the transmission, $\mathbb{T}_{n}^{|m|}(E)$ reaches unity for the electron energy close to the cluster-derived resonance. In so far as the energy of the transmission resonance is determined by the underlying $\mathrm{Na}$ cluster state, it is not surprising that the results reported in Fig. 5 closely resemble those obtained within density-functional formalism for a sodium cluster attached to monatomic sodium chains ${ }^{60}$ Indeed, the coupling of the cluster states to the leads represented by monatomic chains is small, allowing well-defined resonances to be observed.

\section{Cluster formation and conductance}

We are now in a position to explain the difference in the calculated conductance jump from $3 G_{0}$ to $G_{0}$ observed within the $14 a_{0} \leqslant \Delta L \leqslant 21 a_{0}$ elongation range for the cases with five and ten electrons initially in the deformable constriction. The change in the conductance appears over a much broader elongation range in the latter case. As follows from Fig. 3, the conductance variation in this elongation range is determined by the change in transmission probability at the Fermi level for the doubly degenerate ( $m= \pm 1, n=1)$ incident channel. The energy-resolved data presented in Fig. 4 allow us to elucidate the underlying physics.

For the $5 e$ system the transmission probability grows from zero to unity within a narrow energy range above a certain threshold energy $E_{t h}$. Recalling that no clustering happens in the break junction, this is a typical behavior for the change from the under-barrier to over-barrier transmission. The height of the potential barrier separating the two $R=10.7 a_{0}$ leads is determined by the width of the neck formed in the middle of the deformable constriction. For $14 a_{0} \leqslant \Delta L \leqslant 21 a_{0}$ the neck between the leads shrinks (see Fig. 1). The potential barrier and, correspondingly, $E_{t h}$ is raised well above the Fermi level. The transmission at the Fermi level and so the contribution of the $(m= \pm 1, n=1)$ incident channel to the total conductance for the $5 e$ system then sharply drops to zero.

The situation is strikingly different for the $10 e$ system where the $8 e$ cluster is formed in the deformable constriction between the leads. The Fermi level is sandwiched between the 
cluster-localized $p$ and $d$ shells. Transient electron trapping in the cluster-localized states manifests itself through the resonances in the energy-resolved electron transmission through the break junction. According to the discussion above, the transmission is then fixed to unity at the resonance energies below and above the Fermi level. Upon stretching, the clusterlocalized states do not change their energy position, since the underlying cluster structure is preserved. At the same time, since the necks separating the cluster from the $R=10.7 a_{0}$ leads shrink (see Fig. 1), the coupling between the cluster states and leads decreases, causing the resonances to narrow. A dip in the transmission probability gradually develops at $E_{F}$. However, $\mathbb{T}_{1}^{1}\left(E_{F}\right)$ never reaches zero as long as the cluster structure is present in the deformable constriction. Thus, the decrease of the contribution of the $(m= \pm 1, n=1)$ incident channel to the total conductance upon stretching is much softer as compared to the $5 e$ system where it reflects the energy shift of the transmission threshold.

The $8 e$ cluster structure in the break junction disappears for $\Delta L=22 a_{0}$. The $\mathbb{T}_{1}^{1}(E)$ curves then merge for the $5 e$ and $10 e$ systems, reflecting similar geometries of the deformable constrictions where the monatomic wire with magic radius $R=4.5 a_{0}$ is basically formed. As follows from Fig. 4(a) the $\mathbb{T}_{1}^{0}(E)$ is also very similar for the $5 e$ and $10 e$ cases.

For the $m=0$ symmetry, the resonances derived from the $p$ shell and $d$ shell of the $8 e$ cluster in the deformable constriction are energetically well above the potential barrier formed by the necks separating the cluster from the leads. While a dip in the transmission develops between the $s$ - and $p$-shell resonances, as in the $m= \pm 1$ case, the transmission at the Fermi level is nearly unity within the relevant range of elongations. The $(m=0, n=1)$ incident channel then contributes nearly $G_{0}$ to the total conductance.

Coming back to Fig. 2, we observe that the results of Nakamura et al. ${ }^{20}$ also show a rounded shape in the jump from $3 G_{0}$ to $1 G_{0}$, which resembles our $10 e$ curve. This is while no clustering was observed. Nakamura $e t$ al. connect the shape of this jump with the gradual conductance drop of two almost degenerate eigenchannels during smooth atomic displacements. However, in contrast to our $10 e$ system, the calculated in Ref. 20 local density of states integrated over the neck showed wide peaks due to the strong coupling to the leads, i.e., no sharp resonances were found. With respect to the overall length of the conductance curve and, in particular, in the jump from $3 G_{0}$ to $1 G_{0}$, their results are equivalent to our $5 e$ conductance curve.

The above discussion shows that, while the presence of the cluster-derived structures in the break junction is a robust phenomenon as follows from a number of works, ${ }^{23-28,35}$ the particular shape of the conductance change is a much more subtle effect depending on the exact properties of the system at hand. ${ }^{61,62}$ Thus, from the experimental point of view, the evidence for clustering should come from the observation of the resonant structures in transmission. These are directly linked to the very existence of the cluster structures in the break junction. This is possible by measuring the differential conductance, i.e., applying a bias voltage $U$ to the break junction and performing $d I / d U$ spectroscopy upon

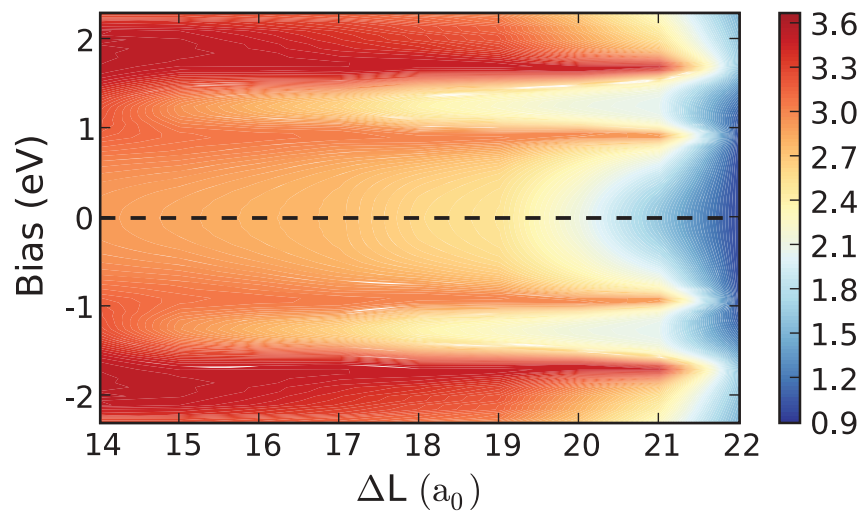

FIG. 6. (Color online) Differential conductance $d I / d U$ map for the breakage of the $R=10.7 a_{0}$ Na nanowire with ten electrons in the deformable construction. The results are shown as a function of the applied bias voltage and nanowire elongation $\Delta L$. The contributions coming from the incident channels $( \pm 1,2)$ and from channels with $|m|>2$ are negligible in the given bias energy range. The horizontal dashed line denotes the zero-bias conductance. For further details see the main text.

elongation. ${ }^{1,40-42}$ That is,

$$
\frac{d I}{d U}=\frac{G_{0}}{2} \sum_{m, n, n^{\prime}}\left[T_{n n^{\prime}}^{m}\left(E_{F}-\frac{U}{2}\right)+T_{n n^{\prime}}^{m}\left(E_{F}+\frac{U}{2}\right)\right],
$$

where the summation runs over the open incident and final channels, and the modification of the deformable constriction by the bias voltage has been neglected. ${ }^{41,42,63}$ The latter approximation should be taken with caution, as the nanowire structure in the break junction can be modified under high bias voltages. $^{64,65}$ The calculated differential conductance is shown in Fig. 6 for the breakage of the $R=10.7 a_{0} \mathrm{Na}$ nanowire with ten electrons in the deformable construction. The clusterderived transmission resonances appear as peak structures in $d I / d U$, as follows from a comparison of Figs. 6 and 4. Thus, the well-defined peak in $d I / d U$ at $\pm 1 \mathrm{eV}$ energy corresponds to the $-0.5 \mathrm{eV}$ resonance within $|m|=1$ symmetry (see Fig.4). The structure at $\pm 1.7 \mathrm{eV}$ arises from the superposition of the resonances at $+0.8 \mathrm{eV}$ within $m=0$ and $|m|=2$ symmetry, and partially, from the broad resonance at $-0.8 \mathrm{eV}$ within $m=0$ symmetry. Observe that the resonant structures remain at fixed energies and sharpen upon elongation. As explained above, the resonance energy is determined by the cluster structure in the break junction, which is particularly stable for the magic clusters. The resonance width depends on the coupling to the leads and thus on the elongation.

Before closing this section, it is worth mentioning that while clustering in the break junction was reported in the previous work by Ogando et al. ${ }^{35}$ no effect of the clusterderived structures on the conductance was observed. For the $R=10.7 a_{0}$ system, those authors obtained three sharp conductance jumps as a function of the nanowire stretching $\Delta L$. We ascribe these qualitatively different results to the quasiclassical Wentzel-Kramers-Brillouin approach used in Ref. 35 for the calculation of the electron transmission through 
the break junction. The present WPP results are exact as long as the potential describing the stretched nanowire is set.

\section{SUMMARY AND CONCLUSIONS}

We have presented a detailed study of the conductance during the elongation and breakage of Na nanowires described with the ultimate jellium model. Self-consistent densityfunctional theory calculations have been performed for the nanowire structure. The resulting one-electron potential has been used then in the follow-up wave packet propagation treatment of the ballistic electron transport through the break junction.

A nanowire of the magic radius $R=10.7 a_{0}$ was studied here. This is a typical example of a stable Na nanowire structure allowing us to address the change of the conductance both in the presence and in the absence of cluster-derived structures in the deformable constriction during the stretching process. Indeed, depending on the size of the deformable constriction, with ten (five) electrons the breakage of the nanowire is (not) preceded by the formation of magic clusters of eight electrons in the break junction. ${ }^{35}$

We observe that the clustering changes the conductance $G$ of the break junction, in particular the shape of the $G=3 G_{0}$ to $G=G_{0}$ step upon elongation. Calculations of the energy- and symmetry-resolved transmission through the break junction reveal the underlying physics. Basically, when a cluster is formed, the electron transport is strongly affected by the transient trapping of ballistic electrons inside the cluster structure. These cluster-localized states sandwich the Fermi level of the $\mathrm{Na}$ leads, and appear as resonances in the transmission of the break junction. It is important that (i) the transmission reaches unity at resonances, and (ii) the energy of the resonances does not change upon elongation as long as a stable "magic" cluster structure is present in the break junction. As a result, the decrease of the conductance upon elongation merely reflects the decrease of the resonance width within the $m=1$ symmetry subspace, i.e., the reduction of the coupling between the cluster and the leads. The conductance $G \simeq 3 G_{0}$ is then maintained over a much broader range of nanowire elongations with a rounded transition to $G=G_{0}$ as compared to the case when no clustering occurs.

For the case when no clustering occurs, the transmission through the break junction shows a threshold behavior. It rapidly grows from zero to unity when the electron energy approaches the height of the potential barrier formed by the neck between the leads. Since the neck shrinks upon elongation, the threshold energy for electrons arriving from the $R=10.7 a_{0}$ leads within the $m=1$ subspace quickly shifts above the Fermi level. This results in an abrupt steplike drop of the conductance from $G=3 G_{0}$ to $G=G_{0}$.

For a realistic system the particular shape of the conductance steps is a subtle phenomenon depending on the number of parameters. At the same time, the present results point at the robust consequence of the presence of a cluster-derived structure in the break junction, which can be used for experimental evidence of clustering. This is the resonant character of the transmission through a break junction with a cluster structure, reflecting transient trapping of the ballistic electron in the cluster-localized states. Thus, a differential conductance study allows us to reveal the cluster-derived resonances and their evolution upon elongation. For the particularly stable "magic" cluster, the resonant structures in the differential conductance maintain their energy within a certain elongation range. This is an indication that the cluster geometry is preserved. The resonance width decreases as a result of the decreasing coupling between cluster structure and leads.

Some comments are in order with respect to the generality of the results presented here. Although the calculations were performed for sodium nanowires, several works point out the possibility of cluster formation for various metals forming a break junction. ${ }^{23-28,35}$ From the point of view of the electronic structure, the energies of the highest occupied molecular orbital and the lowest unoccupied molecular orbital of a finite-size cluster should be close (within a few-eV range) to the Fermi energy of the corresponding metal. It is highly possible that the energy position of the resulting resonances with respect to the Fermi level will resemble that found in the present work, ${ }^{43}$ with similar effects on the conductance and on the differential conductance.

Finally, we hope that this work will stimulate further research on various aspects of cluster formation during contact breaking, such as the probability of appearance of clusters, their stability, and the effect on the conductance.

\section{ACKNOWLEDGMENTS}

This work has been funded partially by the University of the Basque Country UPV/EHU (Grant No. GIC07IT36607), the Departamento de Educación del Gobierno Vasco, and the Spanish Ministerio de Ciencia y Technología (MCyT) (Grant No. FIS2007-66711-C02-01). The SGI/IZO-SGIker UPV/EHU (supported by the National Program for the Promotion of Human Resources within the National Plan of Scientific Research, Development and Innovation - Fondo Social Europeo, MCyT and Basque Government) as well as the Academy of Finland through its Centers of Excellence Program are gratefully acknowledged. We also thank E. Ogando for his collaboration.
${ }^{1}$ N. Agraït, A. Levi Yeyati, and J. M. van Ruitenbeek, Phys. Rep. 377, 81 (2003).

${ }^{2}$ U. Yogeswaran and S.-M. Chen, Sensors 8, 290 (2008).

${ }^{3}$ A. I. Yanson, I. K. Yanson, and J. M. van Ruitenbeek, Nature (London) 400, 144 (1999).

${ }^{4}$ A. I. Yanson, I. K. Yanson, and J. M. van Ruitenbeek, Phys. Rev. Lett. 84, 5832 (2000).
${ }^{5}$ H. Ohnishi, Y. Kondo, and K. Takayanagi, Nature (London) 395, 780 (1998).

${ }^{6}$ G. Rubio, N. Agrait, and S. Vieira, Phys. Rev. Lett. 76, 2302 (1996).

${ }^{7}$ J. M. Krans, J. M. van Ruitenbeek, V. V. Fisun, I. K. Yanson, and L. J. de Jongh, Nature (London) 375, 767 (1995).

${ }^{8}$ J. M. Krans, J. M. van Ruitenbeek, and L. J. de Jongh, Physica B 218, 228 (1996). 
${ }^{9}$ J. Jia, D. Shi, B. Wang, and J. Zhao, Phys. Rev. B 74, 205420 (2006).

${ }^{10}$ W. D. Knight, K. Clemenger, W. A. de Heer, W. A. Saunders, M. Y. Chou, and M. L. Cohen, Phys. Rev. Lett. 52, 2141 (1984).

${ }^{11}$ T. Kizuka, Phys. Rev. Lett. 81, 4448 (1998).

${ }^{12}$ U. Landman, W. D. Luedtke, B. E. Salisbury, and R. L. Whetten, Phys. Rev. Lett. 77, 1362 (1996).

${ }^{13}$ T. N. Todorov and A. P. Sutton, Phys. Rev. Lett. 70, 2138 (1993).

${ }^{14}$ A. M. Bratkovsky, A. P. Sutton, and T. N. Todorov, Phys. Rev. B 52, 5036 (1995).

${ }^{15}$ M. R. Sørensen, M. Brandbyge, and K. W. Jacobsen, Phys. Rev. B 57, 3283 (1998).

${ }^{16}$ E. Z. da Silva, A. J. R. da Silva, and A. Fazzio, Phys. Rev. Lett. 87, 256102 (2001).

${ }^{17}$ P. Z. Coura, S. B. Legoas, A. S. Moreira, F. Sato, V. Rodrigues, S. O. Dantas, D. Ugarte, and D. S. Calvão, Nano Lett. 4, 1187 (2004).

${ }^{18}$ D. Wang, J. Zhao, S. Hu, X. Yin, S. Liang, Y. Liu, and S. Deng, Nano Lett. 7, 1208 (2007).

${ }^{19}$ C. Untiedt, M. J. Caturla, M. R. Calvo, J. J. Palacios, R. C. Segers, and J. M. van Ruitenbeek, Phys. Rev. Lett. 98, 206801 (2007).

${ }^{20}$ A. Nakamura, M. Brandbyge, L. B. Hansen, and K. W. Jacobsen, Phys. Rev. Lett. 82, 1538 (1999).

${ }^{21}$ A. Marchenkov, Z. Dai, C. Zhang, R. N. Barnett, and U. Landman, Phys. Rev. Lett. 98, 046802 (2007).

${ }^{22}$ P. Jelínek, R. Pérez, J. Ortega, and F. Flores, Phys. Rev. B 68, 085403 (2003).

${ }^{23}$ P. Jelínek, R. Pérez, J. Ortega, and F. Flores, Phys. Rev. B 77, 115447 (2008).

${ }^{24}$ R. N. Barnett and U. Landman, Nature (London) 387, 788 (1997).

${ }^{25}$ U. Landman, Solid State Commun. 107, 693 (1998).

${ }^{26}$ H. Häkkinen and M. Manninen, Europhys. Lett. 44, 80 (1998).

${ }^{27}$ L. Hui, F. Pederiva, G. H. Wang, and B. L. Wang, J. Chem. Phys. 119, 9771 (2003).

${ }^{28}$ F. Tavazza, L. E. Levine, and A. M. Chaka, J. Appl. Phys. 106, 043522 (2009).

${ }^{29}$ M. Scheffler and C. Stampfl, in Handbook of Surface Science, edited by K. Horn and M. Schefler (Elsevier, Amsterdam, 2000), Vol. 2, p. 285.

${ }^{30}$ A. Yazdani, D. M. Eigler, and N. D. Lang, Science 272, 1921 (1996).

${ }^{31}$ N. Zabala, M. J. Puska, and R. M. Nieminen, Phys. Rev. Lett. 80, 3336 (1998).

${ }^{32}$ N. Zabala, M. J. Puska, and R. M. Nieminen, Phys. Rev. B 59, 12652 (1999).

${ }^{33}$ C. Yannouleas, E. N. Bogachek, and U. Landman, Phys. Rev. B 57, 4872 (1998).

${ }^{34}$ J. Bürki, C. A. Stafford, and D. L. Stein, Phys. Rev. Lett. 95, 090601 (2005).

${ }^{35}$ E. Ogando, T. Torsti, N. Zabala, and M. J. Puska, Phys. Rev. B 67, 075417 (2003).

${ }^{36}$ M. Manninen, Phys. Rev. B 34, 6886 (1986).

${ }^{37} \mathrm{~J}$. A. Alonso and A. Rubio, in Handbook of Theoretical and Computational Nanotechnology, edited by M. Rieth and W. Schommers
(American Scientific Publishers, Stevenson Ranch, CA, 2006), Vol. 7, Chap. 4, pp. 209-262.

${ }^{38}$ M. Di Ventra, S. T. Pantelides, and N. D. Lang, Phys. Rev. Lett. 84, 979 (2000).

${ }^{39}$ A. Nitzan, Annu. Rev. Phys. Chem. 52, 681 (2001).

${ }^{40}$ A. Nitzan and M. A. Ratner, Science 300, 1384 (2003).

${ }^{41}$ Y. Xue and M. A. Ratner, Phys. Rev. B 68, 115406 (2003).

${ }^{42}$ W. Tian, S. Datta, S. Hong, R. Reifenberger, J. I. Henderson, and C. P. Kubiak, J. Chem. Phys. 109, 2874 (1998).

${ }^{43}$ F. Chen, J. Hihath, Z. Huang, X. Li, and N. J. Tao, Annu. Rev. Phys. Chem. 58, 535 (2007).

${ }^{44}$ R. O. Jones and O. Gunnarson, Rev. Mod. Phys. 61, 689 (1989).

${ }^{45}$ M. Heiskanen, T. Torsti, M. J. Puska, and R. M. Nieminen, Phys. Rev. B 63, 245106 (2001).

${ }^{46}$ A. G. Borisov, A. K. Kazansky, and J. P. Gauyacq, Phys. Rev. B 59, 10935 (1999).

${ }^{47}$ E. V. Chulkov, A. G. Borisov, J. P. Gauyacq, D. Sanchez-Portal, V. M. Silkin, V. P. Zhukov, and P. M. Echenique, Chem. Rev. 106, 4160 (2006).

${ }^{48}$ D. Kosloff and R. Kosloff, J. Comput. Phys. 52, 35 (1983).

${ }^{49}$ D. Neuhauser and M. Baer, J. Phys. Chem. 94, 185 (1990).

${ }^{50}$ M. D. Feit, J. A. Fleck Jr., and A. Steiger, J. Comput. Phys. 47, 412 (1982).

${ }^{51}$ See the review paper by C. Leforestier, R. H. Bisseling, C. Cerjan, M. D. Feit, R. Friesner, A. Guldberg, A. Hammerich, G. Jolicard, W. Karrlein, H. D. Meyer, N. Lipkin, O. Roncero, and R. Kosloff, J. Comput. Phys. 94, 59 (1991); and references therein.

${ }^{52}$ J. Sjakste, A. G. Borisov, J. P. Gauyacq, and A. K. Kazansky, J. Phys. B 37, 1593 (2004).

${ }^{53}$ M. Büttiker, Y. Imry, R. Landauer, and S. Pinhas, Phys. Rev. B 31, 6207 (1985).

${ }^{54}$ M. Tsukada, N. Kobayashi, M. Brandbyge, and S. Nakanishi, Prog. Surf. Sci. 64, 139 (2000).

${ }^{55}$ J. C. Cuevas, A. Levy Yeyati, and A. Martín-Rodero, Phys. Rev. Lett. 80, 1066 (1998).

${ }^{56}$ Y. J. Lee, M. Brandbyge, M. J. Puska, J. Taylor, K. Stokbro, and R. M. Nieminen, Phys. Rev. B 69, 125409 (2004).

${ }^{57}$ P. A. Khomyakov and G. Brocks, Phys. Rev. B 74, 165416 (2006).

${ }^{58}$ H.-W. Lee and C. S. Kim, Phys. Rev. B 63, 075306 (2001).

${ }^{59}$ A. G. Borisov, F. J. Garcia de Abajo, and S. V. Shabanov, Phys. Rev. B 71, 075408 (2005).

${ }^{60}$ R. Gutiérrez, F. Grossmann, O. Knospe, and R. Schmidt, Phys. Rev. A 64, 013202 (2001).

${ }^{61}$ J. C. Cuevas, A. Levy Yeyati, A. Martín-Rodero, G. R. Bollinger, C. Untiedt, and N. Agraït, Phys. Rev. Lett. 81, 2990 (1998).

${ }^{62}$ E. Scheer, N. Agraï, J. C. Cuevas, A. L. Yeyati, B. Ludoph, A. Martín-Rodero, G. R. Bollinger, J. M. van Ruitenbeek, and C. Urbina, Nature (London) 394, 154 (1998).

${ }^{63}$ N. Agraït, C. Untiedt, G. Rubio-Bollinger, and S. Vieira, Phys. Rev. Lett. 88, 216803 (2002).

${ }^{64}$ K. Itakura, K. Yuki, S. Kurokawa, H. Yasuda, and A. Sakai, Phys. Rev. B 60, 11163 (1999).

${ }^{65} \mathrm{C}$. Hu, Y. Gohda, S. Furuya, and S. Watanabe, Sci. Technol. Adv. Mater. 4, 585 (2003). 